(C) 2017 IEEE. Personal use of this material is permitted. Permission from IEEE must be obtained for all other uses, in any current or future media, including reprinting/republishing this material for advertising or promotional purposes, creating new collective works, for resale or redistribution to servers or lists, or reuse of any copyrighted component of this work in other works. 


\title{
Cyclostationary Analysis of Analog Least Mean Square Loop for Self-Interference Cancellation in In-Band Full-Duplex Systems
}

\author{
Anh Tuyen Le, Le Chung Tran, Member, IEEE, and Xiaojing Huang, Senior Member, IEEE
}

\begin{abstract}
Analog least mean square (ALMS) loop is a promising mechanism to suppress self-interference (SI) in an in-band full-duplex (IBFD) system. In this letter, a general solution for the weighting error function is derived to investigate the performance of the ALMS loop employed in any IBFD system. The solution is then applied to IBFD systems with single carrier and multi-carrier signaling respectively. It is shown that due to the cyclostationary property of the transmitted signal, the weighting error function in the multi-carrier system varies more significantly than that in the single carrier one. Therefore, if the ALMS loop can perfectly mimic the SI channel, SI in the single carrier system can be suppressed to a much smaller level than that in the multi-carrier counterpart.
\end{abstract}

Index Terms-In-Band Full-Duplex, self-interference cancellation, and ALMS loop.

\section{INTRODUCTION}

In-band full-duplex (IBFD) transmission is a promising solution to improve the efficiency of frequency spectrum utilization [1]. It is also regarded as a key technology for the next generation mobile broadband networks [2]. To enable IBFD, the most crucial issue is to mitigate the self-interference (SI) caused by the transmitter to its co-located receiver. Among various approaches proposed in the literature, a closed-loop multi-tap filter is proved as the most effective one in the radio frequency (RF) domain especially for wideband applications [3]. This multi-tap adaptive filter can be implemented in the analog domain by employing least mean square (LMS) principle. However, conventional LMS loop requires an integrator which is difficult to build at RF stage. As a result, many existing SI cancellation filters implemented the LMS loop at baseband stage with additional down-conversion circuits [4], [5]. Some others even used dedicated digital modules with sophisticated algorithms to control the weighting coefficients [3], [6]. Obviously, these additional blocks consume more power and produce further noise and interference to the receiver.

An LMS loop purely implemented at RF stage, so called analog LMS (ALMS) loop, was proposed in [7] where a lowpass filter (LPF) was used to replace the ideal integrator. The behaviors of the ALMS loop were investigated by examining the weighting error function. This is the first paper considering the cyclostationary properties of the transmitted signal in regards to the behavior of the cancellation circuit. However, the analysis was only conducted for a single carrier system, and the solution for weighting error function was only derived for the specific root raised cosine (RRC) pulse shaping.

A. T. Le and X. Huang are with Faculty of Engineering and IT, University of Technology Sydney, Australia (emails: anhtuyen.le@student.uts.edu.au and xiaojing.huang@uts.edu.au).

L.C. Tran is with Faculty of Engineering and Information Sciences, University of Wollongong, Australia (email: 1ctran@uow.edu.au).
In this letter, we firstly extend the solution of the weighting error function in a general case. This general solution can be applied to investigate the performance of the ALMS loop employed in any IBFD system. It is revealed that, due to the cyclostationary effects of the transmitted signal, the weighting error function cannot converge to a stable value, but it varies periodically. Consequently, there always exists an irreducible SI whose power depends on the variation of the weighting error function. The solution is then applied to compare the performance of the ALMS loop in a single carrier system to that in a multi-carrier such as an orthogonal frequency division multiplexing (OFDM) system. We show that the convergence speed of the weighting error function is the same in both cases and depends on the loop gain. However, the weighting error function in the single carrier system has a smaller variation than that in the OFDM case. The irreducible interference suppression ratio lower bounds (ISRLBs) are also derived for the two systems respectively. Comparison between them shows that more SI cancellation can be ultimately achieved for the single carrier system. Hence, the main contribution of this letter is the general solution for the weighting error function which can be used as a key to estimate the ultimate level of SI cancellation obtained by the ALMS loop. ISRLB is a very important performance metric in designing the whole IBFD system.

The rest of this letter is organized as follows. Section II describes the mathematical models of the transmitted signals and the structure of the ALMS loop. In Section III, we derive a general solution of the weighting error function for the ALMS loop and compare its performance in single carrier and OFDM systems. Finally, conclusions are drawn in Section IV.

\section{Signal And System Description}

\section{A. Signal Models}

Consider an IBFD system including a baseband part which can operate in either single carrier or OFDM mode and an RF part employing an ALMS loop. The transmitted RF signal is expressed as $x(t)=\operatorname{Re}\left\{X(t) e^{2 \pi f_{c} t}\right\}$ where $f_{c}$ is the carrier frequency, and $X(t)$ is the baseband equivalent which is further denoted as $X_{s}(t)$ in the single carrier mode and $X_{o}(t)$ in the OFDM mode. Mathematically, $X_{s}(t)$ and $X_{o}(t)$ can be expressed as

and

$$
X_{s}(t)=\sum_{i=-\infty}^{\infty} a_{i} p\left(t-i T_{s}\right)
$$

$$
\begin{aligned}
X_{o}(t)= & \sum_{n=-\infty}^{\infty} \sum_{m=-\infty}^{\infty} \sum_{k=-N_{s t} / 2, k \neq 0}^{N_{s t} / 2} a_{k, m} e^{j 2 \pi \frac{k}{N}\left(n-m \frac{T_{o}}{T_{s}}\right)} \\
& \cdot w\left[n-m \frac{T_{o}}{T_{s}}\right] p\left(t-n T_{s}\right)
\end{aligned}
$$


respectively, where $a_{i}$, and $a_{k, m}$ are the $i$-th data symbol in the single carrier system and the data symbol on the $k$ th sub-carrier of the $m$-th OFDM symbol respectively; $T_{s}$ is the symbol period of the single carrier system and also the sample period of the OFDM system; $T_{o}$ is the OFDM symbol period; $N_{s t}$ is the total number of data subcarriers; $N$ is the number of samples in one OFDM symbol excluding cyclic prefix; $w[n]$ is the discrete windowing function applied to an OFDM symbol; and $p(t)$ is the pulse shaping function. The root mean square amplitude of the transmitted signal is defined as $V_{X}=\sqrt{\frac{1}{T} \int_{0}^{T} E\left\{|X(t)|^{2}\right\} d t}$, where $E\{$. stands for expectation; $T$ is the period of transmitted data symbol, i.e., $T_{s}$ or $T_{o}$. The complex data symbols $a_{i}$ and $a_{k, m}$ are assumed to be independent to each other in single carrier and OFDM systems respectively. The autocorrelation function of the transmitted baseband signal $X(t)$ is defined as $\Phi(t, \tau)=E\left\{X^{*}(t) X(t-\tau)\right\}$. With the symbol independence assumption, the autocorrelation functions of single carrier and OFDM signals can be derived as

and

$$
\Phi_{s}(t, \tau)=\sum_{i=-\infty}^{\infty} p^{*}\left(t-i T_{s}\right) p\left(t-\tau-i T_{s}\right)
$$

$$
\begin{aligned}
\Phi_{o}(t, \tau)= & \sum_{l=-\infty}^{\infty} \sum_{l^{\prime}=-\infty k=-N_{s t} / 2, k \neq 0}^{\infty} \sum^{N_{s t} / 2} e^{-j 2 \pi \frac{k}{N}\left(l^{\prime}-l\right)} \\
& \cdot w[l] w\left[l^{\prime}\right] g\left(t-l T_{s},\left(l^{\prime}-l\right) T_{s}+\tau\right)
\end{aligned}
$$

respectively, where $g(t, \tau)=\sum_{m=-\infty}^{\infty} p^{*}\left(t-m T_{o}\right) p\left(t-m T_{o}-\tau\right)$. We see that $\Phi_{s}(t, \tau)=\Phi_{s}\left(t+T_{s}, \tau\right)$ and $\Phi_{o}(t, \tau)=\Phi_{o}\left(t+T_{o}, \tau\right)$ for all $t$ and $\tau$. Therefore, both transmitted signals $X_{s}(t)$ and $X_{o}(t)$ can be treated as wide-sense cyclostationary processes. B. ALMS Loop

The architecture of the ALMS loop proposed in [7] is shown in Fig.1. This is an $L$-stage multi-tap filter in which each tap has

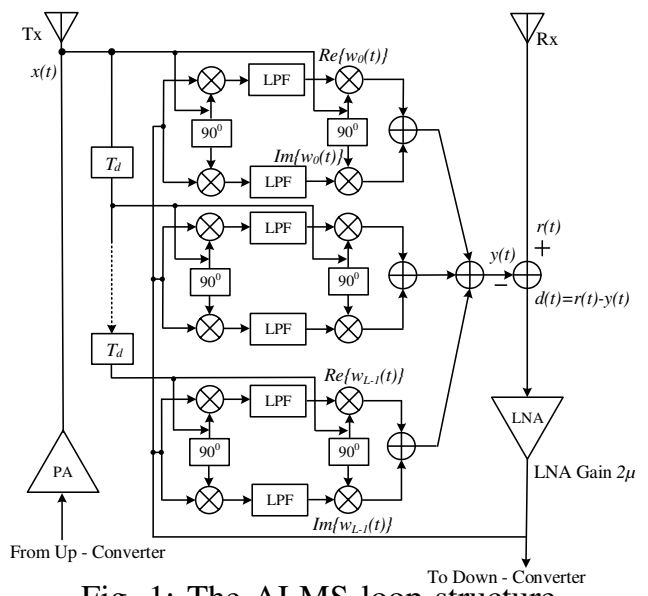

Fig. 1: The ALMS loop structure. a fixed delay $T_{d}$. To avoid spectral overlapping, $T_{d}$ is chosen as $T_{d} \leq T_{s}$. The cancellation signal $y(t)$ is generated to cancel the SI $z(t)$ included in the received signal $r(t)=z(t)+s(t)+n(t)$ where $s(t)$ is the received signal from a remote transmitter, and $n(t)$ is the additional Gaussian noise. The residual signal $d(t)$ is amplified by the low noise amplifier (LNA) and multiplied using the I/Q demodulation architecture with the delayed versions of the transmitted signal $x(t)$. The outputs of the $l$-th I/Q demodulator are filtered by respective Resistor-
Capacitor (RC) LPFs with constant $\alpha(\alpha=1 / R C)$ to generate the complex weight coefficient $w_{l}(t)$ which is derived in [7] as

$$
\begin{array}{r}
w_{l}(t)=\frac{2 \mu \alpha}{K_{1} K_{2}} \int_{0}^{t} e^{-\alpha(t-\tau)}[r(\tau)-y(\tau)] \\
\cdot X\left(\tau-l T_{d}\right) e^{j 2 \pi f_{c}\left(\tau-l T_{d}\right)} d \tau
\end{array}
$$

where $K_{1}$ and $K_{2}$ are the dimensional constants of multipliers in the I/Q demodulator and I/Q modulator respectively; and $2 \mu$ is the gain of the LNA. Assume that the SI channel is modeled as an $L$-stage multi-tap filter where each tap has a coefficient $h_{l}^{*}$ and delay $T_{d}$, and hence the baseband equivalent of the SI $z(t)$ can be expressed as $Z(t)=\sum_{l=0}^{L-1} h_{l}^{*} X\left(t-l T_{d}\right)$. Since the cancellation signal $y(t)$ is constructed as $y(t)=$ $\operatorname{Re}\left\{\sum_{l=0}^{L-1} w_{l}^{*}(t) X\left(t-l T_{d}\right) e^{j 2 \pi f_{c}\left(t-l T_{d}\right)}\right\}$, the performance of the ALMS loop can be determined by the weighting error function $u_{l}(t)=h_{l}-w_{l}(t) e^{j 2 \pi f_{c} l T_{d}}$. Its expected value $\bar{u}_{l}(t)$ is derived in [7] as

$$
\bar{u}_{l}(t)=h_{l}-\frac{\mu \alpha}{K_{1} K_{2}} \int_{0}^{t} e^{-\alpha(t-\tau)} \sum_{l^{\prime}=0}^{L-1} \bar{u}_{l^{\prime}}(\tau) \Phi\left(\tau,\left(l-l^{\prime}\right) T_{d}\right) d \tau \text {. }
$$

This equation shows that the weighting error function not only depends on the loop parameters $\alpha, \mu$ and $K_{1} K_{2}$ but also relates to the autocorrelation function of the transmitted signal, and thus the cyclostationary properties will have significant impact on the ALMS loop performance.

\section{CyClOSTATIONARY ANALYSiS}

\section{A. General Solution of Weighting Error Function}

It is very difficult to solve (6) in a general case. However, if the autocorrelation function of the transmitted signal satisfies that

$$
\Phi(t, \tau)= \begin{cases}V_{X}^{2} \tilde{\Phi}(t, 0), & \text { for } \tau=0 \\ 0 & \text { for } \tau=\text { integer multiples of } T_{d}\end{cases}
$$

where $\tilde{\Phi}(t, 0)$ is the normalized autocorrelation function, (6) can be simplified as

$$
\bar{u}_{l}(t)=h_{l}-\alpha \mu A^{2} \int_{0}^{t} e^{-\alpha(t-\tau)} \bar{u}_{l}(\tau) \tilde{\Phi}(\tau, 0) d \tau
$$

where $A^{2}=V_{X}^{2} / K 1 K 2$. Taking the differentiation with respect to $t$ on both sides of (8), we have

$$
\begin{aligned}
\frac{d \bar{u}_{l}(t)}{d t} & =\mu A^{2} \alpha^{2} \int_{0}^{t} e^{-\alpha(t-\tau)} \bar{u}_{l}(\tau) \tilde{\Phi}(\tau, 0) d \tau-\mu \alpha A^{2} \bar{u}_{l}(t) \tilde{\Phi}(t, 0) \\
& =\alpha\left[h_{l}-\bar{u}_{l}(t)\right]-\mu \alpha A^{2} \bar{u}_{l}(t) \tilde{\Phi}(t, 0)
\end{aligned}
$$

which can be further rearranged in the form of the ordinary differential equation (ODE), i.e.,

$$
\frac{d \bar{u}_{l}(t)}{d t}+\alpha\left[1+\mu A^{2} \tilde{\Phi}(t, 0)\right] \bar{u}_{l}(t)=\alpha h_{l} \text {. }
$$

The solution for the homogeneous form of the ODE, i.e., $U^{\prime}(t)+\alpha\left[1+\mu A^{2} \tilde{\Phi}(t, 0)\right] U(t)=0$ can be found by rearranging it as

$$
\frac{U^{\prime}(t)}{U(t)}=-\alpha\left[1+\mu A^{2} \tilde{\Phi}(t, 0)\right] .
$$

Integrating both sides from 0 to $t$, we get $\ln U(t)=-\alpha \int_{0}^{t}[1+$ $\left.\mu A^{2} \tilde{\Phi}(\tau, 0)\right] d \tau+\ln U(0)$ so that

$$
U(t)=U(0) e^{-\alpha \int_{0}^{t}\left[1+\mu A^{2} \tilde{\Phi}(\tau, 0)\right] d \tau} .
$$

Replacing $U(0)$ by a function $f(t), \quad \bar{u}_{l}(t)=$ $f(t) e^{-\alpha \int_{0}^{t}\left[1+\mu A^{2} \tilde{\Phi}(\tau, 0)\right] d \tau}$ is the solution for the non-homogeneous form of the ODE. Taking the 
differentiation of $\bar{u}_{l}(t)$ and substituting it into (10) we get $f^{\prime}(t)=\alpha h_{l} e^{-\alpha \int_{0}^{t}\left[1+\mu A^{2} \tilde{\Phi}(\tau, 0)\right]} d \tau$. Therefore, $f(t)=\alpha h_{l} \int_{0}^{t} e^{\alpha \int_{0}^{\tau}\left[1+\mu A^{2} \tilde{\Phi}(v, 0)\right] d v} d \tau+C \quad$ where $\quad \mathrm{C}$ is any constant. The solution for $\bar{u}_{l}(t)$ is thus

$$
\begin{aligned}
\bar{u}_{l}(t) & =\left[\alpha h_{l} \int_{0}^{t} e^{\alpha \int_{0}^{\tau}\left[1+\mu A^{2} \tilde{\Phi}(v, 0)\right] d v} d \tau+C\right] \\
& \cdot e^{-\alpha \int_{0}^{t}\left[1+\mu A^{2} \tilde{\Phi}(\tau, 0)\right] d \tau} \\
& =\left[\alpha h_{l} \int_{0}^{t} e^{-\alpha\left(1+\mu A^{2}\right)(t-\tau)} e^{\alpha \mu A^{2} \int_{0}^{\tau}[\tilde{\Phi}(v, 0)-1] d v} d \tau\right. \\
& \left.+C e^{-\alpha\left(1+\mu A^{2}\right) t}\right] e^{-\alpha \mu A^{2} \int_{0}^{t}[\tilde{\Phi}(\tau, 0)-1] d \tau} .
\end{aligned}
$$

When $\alpha \mu A^{2} \int_{0}^{\tau}[\tilde{\Phi}(v, 0)-1] d v \ll 1$ and $t \gg 1 / \alpha\left(1+\mu A^{2}\right)$, $\int_{0}^{t} e^{-\alpha\left(1+\mu A^{2}\right)(t-\tau)} e^{\alpha \mu A^{2} \int_{0}^{\tau}[\tilde{\Phi}(v, 0)-1] d v} d \tau \approx \frac{1}{\alpha\left(1+\mu A^{2}\right)}$. Therefore, $\quad \bar{u}_{l}(t) \approx\left[\frac{h_{l}}{1+\mu A^{2}}+C e^{-\left(1+\mu A^{2}\right) t}\right] e^{-\mu A^{2} \alpha \int_{0}^{t}[\tilde{\Phi}(\tau, 0)-1] d \tau}$. From the initial condition that $\bar{u}_{l}(0)=h_{l}$ we have $C=h_{l} \frac{\mu A^{2}}{1+\mu A^{2}}$, and hence the final solution is

$$
\bar{u}_{l}(t)=\left[h_{l} \frac{1+\mu A^{2} e^{-\alpha\left(1+\mu A^{2}\right) t}}{1+\mu A^{2}}\right] e^{-\mu A^{2} \alpha q(t)}
$$

where $q(t)=\int_{0}^{t}[\tilde{\Phi}(\tau, 0)-1] d \tau$.

Due to the cyclostationary properties of $\tilde{\Phi}(\tau, 0)$, we see that $e^{-\mu \alpha A^{2} q(t)}$ is a periodical function so that $\bar{u}_{l}(t)$ varies periodically. If there was no cyclostationary effect, the weighting error function, denoted as $\breve{u}_{l}(t)$, would have the expectation $E\left\{\breve{u}_{l}(t)\right\}=h_{l} \frac{1+\mu A^{2} e^{-\alpha\left(1+\mu A^{2}\right) t}}{1+\mu A^{2}}$, which would converge to a stable value $h_{l} \frac{1}{1+\mu A^{2}}$ when $t \gg 1 / \alpha\left(1+\mu A^{2}\right)$. In this case the residual SI could be further removed in the digital domain. However, the presence of cyclostationary effect in the residual SI makes it impossible to be completely removed in digital domain. Thus there always exists an irreducible interference whose power $P_{I I}$ is determined by the variation between $u_{l}(t)$ and $\breve{u}_{l}(t)$. The expected value of this variation is denoted as $\tilde{u}_{l}(t)=E\left\{u_{l}(t)-\breve{u}_{l}(t)\right\}=h_{l} \frac{1}{1+\mu A^{2}}\left(e^{-\mu A^{2} \alpha q(t)}-1\right)$ when $t \gg 1 / \alpha\left(1+\mu A^{2}\right)$. Since $E\left\{|X|^{2}\right\} \geq|E\{X\}|^{2}$ for any random process $X$, the time averaged $P_{I I}$ is

$$
\begin{aligned}
P_{I I} & =\frac{A^{2}}{2} \sum_{l=0}^{L-1} \frac{1}{T} \int_{0}^{T} E\left\{\left|u_{l}(t)-\breve{u}_{l}(t)\right|^{2}\right\} d t \\
& \geq \frac{A^{2}}{2} \sum_{l=0}^{L-1} \frac{1}{T} \int_{0}^{T}\left|E\left\{u_{l}(t)-\breve{u}_{l}(t)\right\}\right|^{2} d t=\frac{A^{2}}{2} \sum_{l=0}^{L-1} \frac{1}{T} \int_{0}^{T}|\tilde{u}(t)|^{2} d t \\
& =P_{I} \frac{1}{T} \int_{0}^{T}\left[\frac{1}{1+\mu A^{2}}\left(e^{-\alpha \mu A^{2} q(t)}-1\right)\right]^{2} d t \approx P_{I} \frac{1}{T} \int_{0}^{T}[\alpha q(t)]^{2} d t
\end{aligned}
$$

where $P_{I}=\frac{A^{2}}{2} \sum_{l=0}^{L-1}\left|h_{l}\right|^{2}$ is the normalized interference power. Based on the lower bound of $P_{I I}$, the irreducible interference suppression lower bound (ISRLB) defined as

$$
I S R L B=\frac{P_{I} \frac{1}{T} \int_{0}^{T}[\alpha q(t)]^{2} d t}{P_{I}}=\frac{1}{T} \int_{0}^{T}[\alpha q(t)]^{2} d t
$$

can be used as a measure to compare the performance of the ALMS loop for different types of the transmitted signal. Therefore, ISRLB is an important figure to be considered in the cancellation design process. In the following section, we compare the performance of the ALMS loop in a single carrier system with that in a multi-carrier one to show the impact of cyclostationary properties.

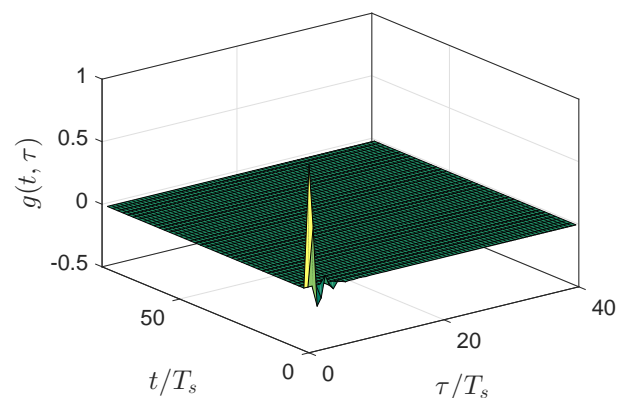

Fig. 2: One period of $g(t, \tau)$ with $T_{o}=80 T_{s}$.

\section{B. Single Carrier Versus OFDM}

To apply the above solution of the weighting error function of the ALMS loop to the two systems, we firstly examine their respective autocorrelation functions. For a single carrier system with RRC pulse shaping function, it is shown in [7] that $\Phi_{s}(t, \tau)$ satisfies (7) with a closed-form as

$$
\Phi_{s}(t, \tau) \approx \begin{cases}V_{X}^{2}\left(\frac{2 \beta_{s}}{\pi} \cos \frac{2 \pi t}{T_{s}}+1\right), & \text { for } \tau=0 \\ 0, & \text { for } \tau=\text { integer multiples of } T_{s}\end{cases}
$$

where $\beta_{s}$ is the roll-off factor of the RRC pulse shaping function. Hence, $q(t)$ for the single carrier system is derived as $q_{s}(t)=T_{s} \frac{\beta_{s}}{\pi^{2}} \sin \frac{2 \pi t}{T s}$ [7, Eq.(17)]. In case of the multi-carrier system, an IEEE802.11a baseband is taken as an example. We firstly examine the autocorrelation $g(t, \tau)$ of the pulse shaping function $p(t)$ introduced in (4), which is a periodical function of the period $T_{o}$. One period of $g(t, \tau)$ with the power of $p(t)$ normalized to 1 is shown in Fig. 2. Obviously, $g(t, \tau) \approx 0$ when $\tau$ is any integer multiple of $T_{s}$, and hence the autocorrelation function of this OFDM signal at $\tau=0$ becomes $\Phi_{o}(t, 0)=N_{s t} \sum_{l=-\infty}^{\infty} w^{2}[l] g\left(t-l T_{s}, 0\right)$ with the period $T_{o}$. For simplicity, one period of the convolution of $w^{2}[l]$ and $g(t, 0)$ can be further approximated as a continuous window $w^{2}(t)$. Therefore, the autocorrelation function of the OFDM signal has a closed form of a periodical function of $t$ whose period contains the continuous window $w^{2}(t)$, i.e.,

$$
\Phi_{o}\left(t,\left(l-l^{\prime}\right) T_{d}\right) \approx \begin{cases}V_{X}^{2} \sum_{m=-\infty}^{\infty} w^{2}\left(t-m T_{o}\right), & \text { for } l=l^{\prime} \\ 0, & \text { for } l \neq l^{\prime}\end{cases}
$$

where $w(t), 0 \leq t \leq T_{o}$, is the normalized continuous windowing function such that $\frac{1}{T_{o}} \int_{0}^{T_{o}} w^{2}(t) d t=1$. For the discrete windowing function recommended in the IEEE802.11a standard [8], after conversion to the continuous function and normalization, we have expression of the windowing function $w(t)$ as

$$
w(t)=\sqrt{\frac{4\left(1+\beta_{o}\right)}{4-\beta_{o}}} \begin{cases}\sin ^{2}\left(\frac{\pi}{2}\left(\frac{t}{T_{1}}\right)\right) & 0 \leq t<T_{1} \\ 1 & T_{1} \leq t<T_{2} \\ \sin ^{2}\left(\frac{\pi}{2}\left(\frac{T_{o}-t}{T_{1}}\right)\right) & T_{2} \leq t<T_{o}\end{cases}
$$

where $T_{1}=\beta_{o} T_{o} /\left(1+\beta_{o}\right)$ and $T_{2}=T_{o} /\left(1+\beta_{o}\right)$ with $\beta_{o}$ as the roll-off factor of the windowing function. Applying the above solution, we find the OFDM version of the $q(t)$ function as

$$
q_{o}(t)= \begin{cases}\frac{5\left(\beta_{o}-1\right)}{2\left(4-\beta_{o}\right)} t-\frac{2 \beta_{o} T_{o}}{\left(4-\beta_{o}\right) \pi} \sin \left(\frac{\pi t}{T_{1}}\right)+ & 0 \leq t<T_{1} \\ \frac{\beta_{o} T_{o}}{4 \pi\left(4-\beta_{o}\right)} \sin \left(\frac{2 \pi t}{T_{1}}\right) & T_{1} \leq t<T_{2} \\ \frac{5 \beta_{o}}{4-\beta_{o}}\left(t-T_{o} / 2\right) & \\ \frac{5\left(\beta_{o}-1\right)}{2\left(4-\beta_{o}\right)}\left(t-T_{o}\right)+\frac{2 \beta_{o} T_{o}}{\left(4-\beta_{o}\right)} \sin \left(\frac{\pi\left(T_{o}-t\right)}{T_{1}}\right) & \\ -\frac{\beta_{o} T_{o}}{4 \pi\left(4-\beta_{o}\right)} \sin \left(\frac{2 \pi\left(T_{o}-t\right)}{T_{1}}\right) & T_{2} \leq t<T_{o} .\end{cases}
$$




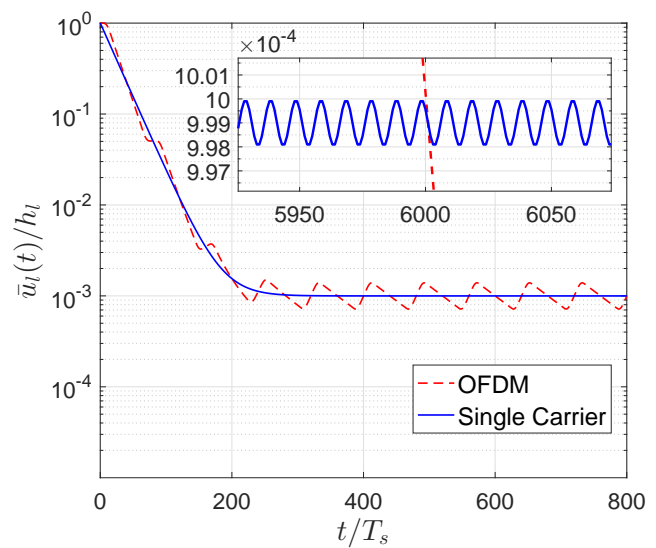

Fig. 3: Normalized weighting error functions with the loop gain $\mu A^{2}=1000, \alpha T_{s}=0.003, T_{o}=80 T_{s}$, and $\beta_{s}=\beta_{o}=0.25$.

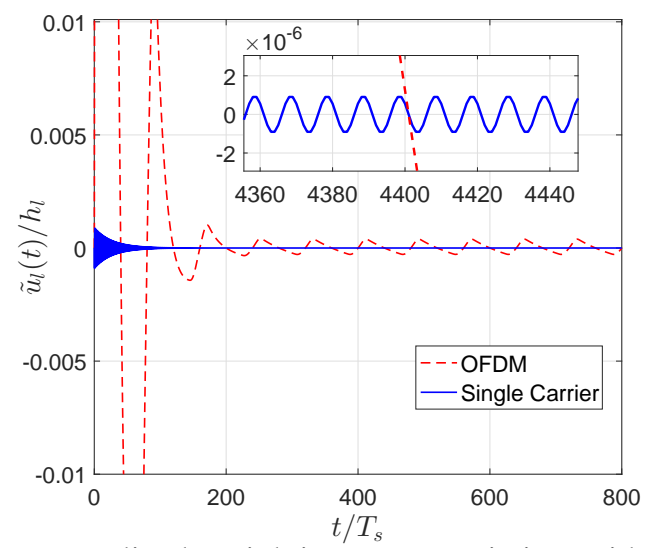

Fig. 4: Normalized weighting error variation with the loop gain $\mu A^{2}=1000, \alpha T_{s}=0.003, T_{o}=80 T_{s}$, and $\beta_{s}=\beta_{o}=0.25$.

From $q_{s}(t), q_{o}(t)$, and (14) we can obtain the weighting error functions for the single carrier and OFDM systems as $\bar{u}_{l, s}(t)$ and $\bar{u}_{l, o}(t)$ respectively. To compare the performance of the ALMS loop in the two systems, the convergence curve of the normalized weighting error function $\bar{u}_{l}(t) / h_{l}$ for the two cases under loop gain $\mu A^{2}=1000, \alpha T_{s}=0.003$ and $T_{o}=80 T_{s}$ are plotted in Fig. 3. The normalized variation $\tilde{u}_{l}(t) / h_{l}$ is presented in Fig. 4. The insets in Fig. 3 and Fig. 4 show a closer look for the $\bar{u}_{l, s}(t)$ and $\tilde{u}_{l, s}(t)$ respectively.

From Fig. 3, it can be concluded that with the same loop gain $\mu A^{2}$ and the RC constant $\alpha$, the convergence speeds of $\bar{u}_{l, s}(t)$ and $\bar{u}_{l, o}(t)$ are the same for both cases of the transmitted signals. Moreover, both $\bar{u}_{l, s}(t)$ and $\bar{u}_{l, o}(t)$ do not converge to a stable value, but they vary with periods $T_{s}$ and $T_{o}$ respectively. In terms of variation, as shown in Fig. 4, $\tilde{u}_{l, s}(t)$ varies in a smaller range than $\tilde{u}_{l, o}(t)$ does. Thus, the ISRLB of the single carrier system is expected to be smaller than that of the OFDM counterpart. Substituting $q_{s}(t)$ and $q_{o}(t)$ into (16), we obtain the ISRLB for the single carrier and OFDM systems as $I S R L B_{s}=\frac{1}{2}\left(\alpha T_{s} \frac{\beta_{s}}{\pi^{2}}\right)^{2}$, and

$I S R L B_{o}=\frac{\alpha^{2} T_{o}^{2} \beta_{o}^{2}}{\left(4-\beta_{o}\right)^{2}\left(1+\beta_{o}\right)^{2}}\left\{\frac{25}{12}\left(1-\beta_{o}\right)^{2}+\frac{5 \beta_{o}}{16 \pi^{2}}\left(81-55 \beta_{o}\right)\right\}$

respectively. Putting the $I S R L B_{s}$ and $I S R L B_{o}$ together in Fig. 5 as functions of $\alpha T_{s}$ and various values of the roll-off factors $\beta_{s}, \beta_{o}$, we see that, with the same value of $\beta_{s}$ and $\beta_{o}$

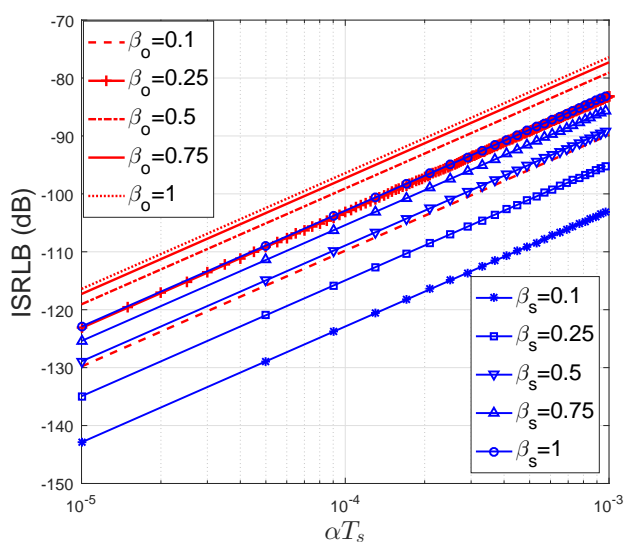

Fig. 5: ISRLB of the two systems with various values of $\beta_{s}$ and $\beta_{o}$.

except for $\beta_{s}=0$ and $\beta_{o}=0, I S R L B_{s}$ is much smaller than $I S R L B_{o}$. It means that when the ALMS loop has exactly the same tap spacing as the SI channel, the SI in the single carrier system can be suppressed to a much lower level than that in the ODFM system. The reason is that the weighting coefficients of the ALMS loop are affected by the autocorrelation function of the transmitted signal as we have analyzed. As the period of an OFDM symbol is much longer than that of a data symbol in the single carrier system, the weight coefficients in the OFDM system vary more significantly.

\section{CONCLUSIONS}

The general solution for the weighting error function is derived to reveal the significant impacts of cyclostationary properties of the transmitted signal on the performance of the ALMS loop. Applying this solution to both single carrier and OFDM IBFD systems, we show that, given the same loop gain and other parameters, the SI can be potentially canceled more effectively to a smaller level of ISRLB in the single carrier system than that in the OFDM system due to the different cyclostationary properties of the transmitted signals. Determining the ISRLB is an important consideration in the SI cancellation design process.

\section{ACKNOWLEDGMENT}

This work was supported by the Australian Research Council (DP160101693).

\section{REFERENCES}

[1] A. Sabharwal et al., "In-band full-duplex wireless: Challenges and opportunities," IEEE J. Sel. Areas Commun, vol. 32, no. 9, pp. 1637$1652,2014$.

[2] S. Han et al., "Full duplex: Coming into reality in 2020?" in 2014 IEEE Global Communications Conference, Dec 2014, pp. 4776-4781.

[3] D. Korpi et al., "Full-duplex mobile device: Pushing the limits," IEEE Communications Magazine, vol. 54, no. 9, pp. 80-87, Sept 2016.

[4] T. Huusari et al., "Wideband self-adaptive RF cancellation circuit for full-duplex radio: Operating principle and measurements," in IEEE 81st Vehicular Technology Conference (VTC Spring), May 2015.

[5] J. Kim et al., "Full-duplex Radios in 5G: Fundamentals, Design and Prototyping," in Signal Processing for 5G. Chichester, UK: John Wiley \& Sons, Ltd, Aug 2016.

[6] K. E. Kolodziej, J. G. McMichael, and B. T. Perry, "Multitap RF canceller for in-band full-duplex wireless communications," IEEE Trans. Wireless Commun., vol. 15, no. 6, pp. 4321-4334, June 2016.

[7] X. Huang and Y. J. Guo, "Radio frequency self-interference cancellation with analog least mean-square loop," IEEE Trans. Microw. Theory Tech., vol. 65, no. 9, pp. 3336-3350, Sept 2017.

[8] "IEEE Wireless LAN Medium Access Control (MAC) and Physical Layer (PHY) Specifications,” IEEE Std 802.11-1997, 1997. 